\title{
Escherichia coli enterotoxigénica y enteroagregativa: prevalencia, patogénesis y modelos múridos
}

Diana Ríos-Muñiz, ${ }^{1,2}$ Jorge Francisco Cerna-Cortés, ${ }^{1}$ Nadia Morán-García, ${ }^{2}$ Mario Meza-Segura ${ }^{2}$ y Teresa Estrada-García *

${ }^{1}$ Departamento de Microbiología, Escuela Nacional de Ciencias Biológicas, Instituto Politécnico Nacional; ${ }^{2}$ Departamento de Biomedicina Molecular, Centro de Investigación y de Estudios Avanzados del Instituto Politécnico Nacional. Ciudad de México, México

\section{Resumen}

Los patotipos de Escherichia coli enterotoxigénica (ETEC) y enteroagregativa (EAEC) son importantes agentes etiológicos causantes de diarrea en niños menores de cinco años de México y países en desarrollo, en quienes causan numerosas muertes. Ambos se han asociado con retraso en el crecimiento infantil y son los principales agentes causales de la "diarrea del viajero". La patogénesis de ambas bacterias se inicia cuando estas se adhieren al epitelio intestinal mediante fimbrias, denominadas factores de colonización en las cepas ETEC aisladas de humano y fimbrias de adherencia agregativa en las cepas de EAEC. Una vez que ETEC se adhiere al enterocito produce una o ambas de sus toxinas e induce la secreción de iones de cloruro, sodio y agua al lumen intestinal, produciendo su característica diarrea acusa. EAEC se une al epitelio intestinal formando una biopelícula, induce la producción de moco, libera sus toxinas y promueve inflamación. Modelos de infección de EAEC y ETEC con ratones C57BL/6 silvestres y deficientes del ligando de CD40 (con microbiotas intactas), respectivamente, revelaron que la desnutrición y la dieta baja en cinc incrementan la infección de EAEC causando retraso en el crecimiento y que ETEC coloniza, persiste e induce respuesta inmune humoral local y sistémica.

PALABRAS CLAVE: Escherichia coli enterotoxigénica. Escherichia coli enteroagregativa. Diarrea infantil. Desnutrición. Modelos múridos.

\section{Abstract}

Enterotoxigenic (ETEC) and enteroaggregative Escherichia coli (EAEC) pathotypes are important etiological agents causative of diarrhea in children younger than 5 years of age in Mexico and in developing countries, where they cause numerous deaths. Both have been associated with delayed growth in children and are the main causative agents of traveler's diarrhea. The pathogenesis of both bacteria starts by adhering to the intestinal epithelium by means of fimbriae, called colonization factors in human ETEC isolates and aggregative adherence fimbriae in EAEC isolates. Once ETEC adheres to the enterocyte, it produces one or both of its toxins and induces the secretion of chloride and sodium ions and water into the intestinal lumen, producing its characteristic watery diarrhea. EAEC binds to the intestinal epithelium forming a biofilm, induces the production of mucus, releases its toxins and promotes inflammation. EAEC and ETEC infection models with wild-type C57BL/6 and CD40 ligand-deficient mice (with intact microbiota), respectively, revealed that undernutrition and low-zinc diet increases EAEC infection, causing growth retardation, and that ETEC colonizes, persists and induces local and systemic humoral immune response.

KEY WORDS: Enterotoxigenic Escherichia coli. Enteroaggregative Escherichia coli. Diarrhea in children. Undernutrition. Murine models.

Correspondencia:

*Teresa Estrada-García

E-mail: testrada@cinvestav.mx
Fecha de recepción: 28-09-2018

Fecha de aceptación: 24-01-2019

DOI: 10.24875/GMM.19004716
Gac Med Mex. 2019;155:410-416

Disponible en PubMed www.gacetamedicademexico.com 


\section{Introducción}

En México, las enfermedades diarreicas son la segunda causa de morbilidad en niños menores de cinco años. ${ }^{1}$ Estas enfermedades presentan una tasa de mortalidad en menores de un año de 27.78 y en niños de uno a cuatro años de 3.47 por cada 100000 niños. ${ }^{2}$ Después de la introducción de la vacuna contra el rotavirus en 2007 , la mortalidad debida a diarrea ha disminuido, ${ }^{3}$ sin embargo, las tasas de morbilidad no se han modificado significativamente.

En México, ${ }^{4,5}$ al igual que en el resto del mundo, se reconoce que los patotipos diarrogénicos de Escherichia coli (PDE) son los principales agentes etiológicos causantes de diarrea en los niños. ${ }^{6-11}$ Hasta el momento, con base en sus características de patogenicidad, se reconocen seis categorías: ${ }^{11}$

- Escherichia coli enteropatógena típica y atípica.

- Escherichia coli enterotoxigénica (ETEC).

- Escherichia coli enteroagregativa (EAEC).

- Escherichia coli enterodifusa.

- Escherichia coli productora de toxinas Shiga.

- Escherichia coli enteroinvasora.

Desafortunadamente, por lo general no se realiza la búsqueda intencionada de los PDE, ya que las pruebas bioquímicas tradicionales solo indican la especie, por lo que la caracterización se lleva a cabo por métodos moleculares mediante la identificación de los diferentes loci de cada patotipo..$^{12,13}$

En México, después de la introducción de la vacuna contra el rotavirus, en un estudio de cuatro años de búsqueda intencionada de patógenos causantes de diarrea aguda en niños que requirieron hospitalización,,$^{14}$ se observó que los PDE (30.9\%) son los patógenos más frecuentemente identificados en los casos de diarrea, por arriba de rotavirus (22.6\%), Salmonella enterica (11.4\%), Shigella spp. (10.8\%), Campylobacter spp. (5.6\%), parásitos (4.2\%) y Vibrio cholerae (1.1\%). ETEC y EAEC están entre los agentes causales más importantes de diarrea e infecciones, principalmente en niños de regiones de ingresos bajos e intermedios, ${ }^{6-9}$ incluyendo México, ${ }^{4,5,14-17} \mathrm{como}$ se muestra en la Tabla 1. Además, se ha registrado que en México las cepas de PDE son resistentes a los antibióticos de primera elección para tratamiento de diarrea aguda grave., ${ }^{4,15}$

Las cepas de ETEC que producen enfermedad en humanos no se aíslan de animales ni producen enfermedad y viceversa, por lo que se consideran especie-específicas,$^{18}$ mientras que las cepas de EAEC solo han sido aisladas en humanos. ${ }^{19}$ De tal forma que hasta el momento se conoce poco acerca de la patogénesis in vivo e in situ de estos dos patotipos, en parte debido a la falta de modelos animales de infección con cepas de ETEC y EAEC aisladas de humano, adecuados y accesibles.

La ventaja de utilizar modelos de ratón es que se cuenta con un gran número de reactivos comerciales contra una gran diversidad de moléculas múridas. Esperamos que los modelos múridos de EAEC y ETEC permitan establecer la respuesta inmune que inducen en el hospedero y el daño que producen, así como las moléculas bacterianas que inducen respuestas protectoras para utilizarlas en el desarrollo de vacunas. Por ello, los objetivos de esta revisión es describir brevemente la epidemiología de estos dos patotipos en general y en particular en México, su patogénesis y el estatus del desarrollo de modelos múridos contra estos patógenos.

\section{Epidemiología de ETEC}

Este patotipo es un importante agente causal de diarrea, responsable de numerosos casos y muertes en niños menores de cinco años de regiones del mundo con ingresos intermedios y bajos; se estima que produce mil millones de casos de diarrea al año $0^{8-10} y$ la mortalidad atribuible en este grupo etario en 2015 fue de 23600 (9600 a 44 300, $95 \%$ de intervalo de incertidumbre). ${ }^{20}$

ETEC también afecta a la población adulta, ya que es el principal agente etiológico, seguido de cerca por EAEC, que produce diarrea en viajeros adultos de países industrializados que visitan zonas endémicas de ETEC y EAEC, denominada "diarrea del viajero" ${ }^{21,22}$ Aproximadamente 3 a $17 \%$ de los adultos con diarrea del viajero posteriormente desarrollan enfermedad inflamatoria intestinal. ${ }^{22}$

En México, con métodos moleculares nuestro equipo de trabajo y otros investigadores hemos demostrado la prevalencia de ETEC en diferentes regiones del país, particularmente en niños ${ }^{4,5,14-17}$ (Tabla 1), pero también en adultos viajeros que visitan México. ${ }^{23}$

Debido a que ETEC causa alta mortalidad en niños de zonas endémicas ${ }^{9,20}$ y a que se ha asociado con retraso en su crecimiento (stunting), ${ }^{24}$ la Organización Mundial de la Salud, por medio del Product Development for Vaccines Advisory Committee, considera una prioridad el desarrollo de vacunas contra este patógeno, ya que una de sus metas para 2025 es reducir la tasa de muertes por neumonía y diarrea 
Tabla 1. Prevalencia de patotipos diarreogénicos de Escherichia coli en diferentes estudios realizados en México

\begin{tabular}{|c|c|c|c|c|c|c|c|c|c|c|c|c|}
\hline \multirow[t]{2}{*}{ Región } & \multicolumn{8}{|c|}{ Diarrea aguda que requirió hospitalización } & \multicolumn{4}{|c|}{ Diarrea de comunidad } \\
\hline & \multicolumn{2}{|c|}{$\begin{array}{l}\text { Ciudad de } \\
\text { México- } \\
\text { Tabasco }^{4}\end{array}$} & \multicolumn{2}{|c|}{$\begin{array}{l}\text { Ciudad de } \\
\text { México }^{17}\end{array}$} & \multicolumn{2}{|c|}{ Yucatán 5} & \multicolumn{2}{|c|}{ Yucatán ${ }^{14}$} & \multicolumn{2}{|c|}{$\begin{array}{c}\text { Comunidad } \\
\text { periurbana }{ }^{16} \\
\text { (Ciudad de México) }\end{array}$} & \multicolumn{2}{|c|}{ Sinaloa ${ }^{15}$} \\
\hline Periodo & \multicolumn{2}{|c|}{$\begin{array}{l}\text { Marzo 2000- } \\
\text { febrero } 2001\end{array}$} & \multicolumn{2}{|c|}{$\begin{array}{l}\text { Septiembre 2004- } \\
\text { diciembre } 2006\end{array}$} & \multicolumn{2}{|c|}{$\begin{array}{c}\text { Enero 2007-2009, } \\
\text { Enero 2010-diciembre } 2011\end{array}$} & \multicolumn{2}{|c|}{$\begin{array}{l}\text { Enero } 2010 \text { - } \\
\text { julio } 2014\end{array}$} & \multicolumn{2}{|c|}{$\begin{array}{l}\text { Enero- } \\
\text { diciembre } 1998\end{array}$} & \multicolumn{2}{|c|}{$\begin{array}{l}\text { Enero 2011- } \\
\text { diciembre } 2014\end{array}$} \\
\hline \multirow[t]{2}{*}{ Pacientes } & \multicolumn{2}{|c|}{$\begin{array}{l}\text { Niños }<5 \\
\text { años }\end{array}$} & \multicolumn{2}{|c|}{$\begin{array}{c}\text { Niños }>2 \mathrm{a}<12 \\
\text { años }\end{array}$} & \multicolumn{2}{|c|}{ Niños $<5$ años } & \multicolumn{2}{|c|}{$\begin{array}{l}\text { Niños }<10 \\
\text { años }\end{array}$} & \multicolumn{2}{|c|}{ Niños $<2$ años } & \multicolumn{2}{|c|}{$\begin{array}{l}\text { Niños, adultos y } \\
\text { ancianos }\end{array}$} \\
\hline & $\mathrm{n}$ & $\%$ & $\mathrm{n}$ & $\%$ & n & $\%$ & n & $\%$ & $\mathrm{n}$ & $\%$ & $\mathrm{n}$ & $\%$ \\
\hline $\begin{array}{l}\text { Núm. de } \\
\text { pacientes }\end{array}$ & 430 & & 300 & & 831 & & 893 & & $173^{*}$ & & 1037 & \\
\hline $\begin{array}{l}\text { PDE } \\
\text { identificado }\end{array}$ & 62 & 14 & 97 & 32.3 & 232 & $27.9^{* \star *}$ & 276 & 31 & 32 & 27 & 242 & 23.3 \\
\hline ETEC & 17 & 26 & 40 & 41.2 & 21 & 9 & 35 & 12.7 & 12 & 37.2 & 43 & 4.3 \\
\hline EAEC & 16 & 26 & - & & 56 & 24 & 56 & 20.3 & - & & 126 & 12.2 \\
\hline EPEC & 16 & 26 & 28 & 28.8 & 46 & 20 & 49 & 17.7 & 16 & 50 & 53 & 5.1 \\
\hline STEC & 11 & 18 & 26 & 26.8 & 1 & 0.4 & 0 & 0 & 1 & 3.1 & 3 & 0.3 \\
\hline DAEC & - & & - & & 81 & 35 & 101 & 36.6 & - & & 15 & 1.4 \\
\hline EIEC & 2 & 3 & 3 & 3 & 1 & 0.4 & 2 & 0.7 & 3 & 9.3 & 2 & 0.2 \\
\hline Mixta** & 0 & 0 & 0 & 0 & 23 & 10 & 33 & 12 & 0 & 0 & 0 & 0 \\
\hline
\end{tabular}

en niños menores de cinco años a menos de uno por cada 1000 nacimientos vivos. ${ }^{20} \mathrm{El}$ impacto de desarrollar vacunas entéricas ha quedado muy claro con la introducción de la vacuna de rotavirus en los esquemas de vacunación infantil, ya que se estima que las muertes atribuibles a diarrea en niños menores de cinco años en México disminuyeron $34 \%$ entre 2005 y $2015 .^{3}$

\section{Patogénesis de ETEC}

La patogénesis de ETEC, como la de cualquier microorganismo, requiere la colonización o infección y la posterior liberación de sus mediadores para que actúen sobre la célula del hospedero. ETEC se define por la producción de las toxinas termolábil (LT) y termoestable (ST), codificadas en plásmidos cuya estructura y mecanismos de acción están bien establecidos. ${ }^{25,26}$ La adherencia de ETEC a los enterocitos está mediada por fimbrias, las cuales también están codificadas en plásmidos; en las cepas ETEC de humanos, estas fimbrias se denominan factores de coIonización. Hasta el momento, en diferentes cepas de
ETEC se han descrito más de 30 factores de colonización y son estas fimbrias las que le confieren a ETEC su especificidad de especie. ${ }^{27}$

También se ha descrito una adhesina denominada etpA, glucoproteína secretada por un sistema de secreción de dos componentes denominado etpBAC, con una función muy particular ya que forma un puente molecular entre las regiones altamente conservadas de la flagelina presentes en la punta del flagelo de ETEC y la superficie celular del huésped. Además, en modelos múridos se ha demostrado que etpA es necesaria para la colonización del intestino delgado y en líneas celulares epiteliales y para la adecuada adhesión y liberación de la toxina LT. ${ }^{25,26}$

Una vez que ETEC se une a la mucosa intestinal libera sus toxinas, que resulta en diarrea acuosa parecida a la del cólera, característica de este patógeno. ${ }^{25,26}$ La toxina LT está estrechamente relacionada en estructura y antigenicidad con la toxina de cólera, tiene una masa molecular de $84 \mathrm{kDa}$ y está constituida por dos subunidades: la subunidad activa (A), rodeada de cinco subunidades $B$ idénticas, las cuales están unidas. ${ }^{25}$ Por su parte, la toxina ST es un péptido de bajo 
peso molecular constituido por 18 o 19 aminoácidos y presenta dos variantes, STp y STh, nombradas así ya que la primera se describió en cerdos y la segunda en humanos. Ambas toxinas finalmente fosforilan el canal CFTR (cystic fibrosis transmembrane regulator channel), estimulando la secreción de iones cloruro, lo cual conlleva la salida de sales y agua del enterocito hacia el lumen intestinal. ${ }^{25}$ La producción de cualquiera de estas dos toxinas es suficiente para causar diarrea severa y los estudios epidemiológicos han mostrado que las cepas de ETEC pueden producir la toxina LT, la toxina ST o ambas. ${ }^{4-10,14-17}$ Se han descrito otros factores de patogenicidad, sin embargo, la mayoría de estos solamente se han descrito en la cepa prototipo. Hasta el momento solo se ha descrito en cepas de ETEC al autotransportador con actividad de serin proteasa denominado EatA, que parece acelerar la salida de LT puesto que degrada a MUC2, la principal mucina secretada por células caliciformes del intestino, y modular la adhesión mediada por etpA. ${ }^{26}$

\section{Modelos múridos de ETEC}

Los modelos múridos se han convertido en una herramienta esencial para entender la compleja interacción entre los microorganismos entéricos con su hospedero y la enfermedad. Debido a la importancia de la microbiota intestinal, ${ }^{28,29}$ el desarrollo de modelos múridos de cualquier patógeno entérico debe incluir una microbiota residente intacta, ya que en ellos se ha mostrado que los antibióticos modifican la microbiota residente del tracto intestinal, incluso los que no son de amplio espectro, lo que altera la disponibilidad de carbohidratos en la mucosa intestinal, que es aprovechada por patógenos intestinales como Salmonella enterica serovar typhimurium y Clostridium difficile. ${ }^{28}$ Además, recientemente se ha demostrado que la composición del microbioma de los ratones, tanto los silvestres como los utilizados en modelos de enfermedad, varía dependiendo del bioterio y esto tal vez explique la poca reproducibilidad de modelos múridos en diferentes laboratorios. ${ }^{29}$

Desde hace varias décadas se ha intentado desarrollar un modelo múrido con la cepa prototipo de ETEC H10407 (serotipo O78:H11), aislada de un paciente adulto con diarrea severa de Bangladesh. Esta cepa contiene dos plásmidos, uno que codifica para la toxina LT y otro para la toxina ST, este último plásmido también codifica para el primer factor de colonización descrito, el cual fue denominado CFA/l, por lo que se conoce como plásmido ST-CFA/I.
El grupo de trabajo del doctor Fleckestein ${ }^{30}$ ha utilizado con éxito ratones de la cepa CD1 para estudiar la colonización de la cepa silvestre ETEC H10407 y de sus mutantes de It y etpA tan solo por 24 horas posinfección. En realidad, en estos estudios se ha utilizado el intestino delgado de los ratones como un tubo de ensayo, ya que antes de la inoculación con las cepas silvestre o mutantes, al igual que en muchos otros estudios, los ratones reciben antibióticos de amplio espectro, lo cual provoca la eliminación de la microbiota intestinal. Recientemente se utilizó un modelo múrido con un ratón C57BL/6 para evaluar el efecto de cinc sobre la infección con ETEC H10407, tanto en animales con dietas normales como con dietas bajas en proteína y deficientes en cinc, sin embargo, antes de la inoculación oral de ETEC los animales fueron tratados con antibióticos, lo que alteró la microbiota intestinal. ${ }^{24}$

Por lo anterior, se deben desarrollar modelos múridos que mantengan la microbiota intacta en los que sea posible evaluar in vivo e in situ la interacción de ETEC con el epitelio intestinal, su colonización y persistencia. En 2013, reportamos por primera vez un modelo múrido de infección y persistencia con la cepa de ratón $\mathrm{C} 57 \mathrm{BL} / 6$ deficiente del ligando de CD40 (C57- $\mathrm{cd} 4 \mathrm{OH}^{-/}$) y ETEC $\mathrm{H} 10407$ (contiene los plásmidos LT y ST-CFA/I), la cual se inoculó oralmente sin previo tratamiento con antibióticos..$^{18}$ Demostramos que los ratones deficientes del ligando permanecieron colonizados por más de 15 días posinfección comparados con los ratones silvestres (C57BL/6), los cuales eliminaron ETEC a los tres días posinfección. Tanto los ratones silvestres como los $\mathrm{C} 57$ - $\mathrm{cd}_{40} \mathrm{O}^{- \text {- }}$ fueron capaces de producir anticuerpos en suero y heces de las clases IgM, IgG e IgA específicos contra ETEC, pero en concentraciones significativamente menores en los ratones $\mathrm{C} 57-\mathrm{Cd}_{40 \mathrm{O}^{-}}$. Con el fin de evaluar el papel de los plásmidos ETEC H10407 en la infección, se inocularon oralmente a grupos de ratones silvestre y C57-cd40\%- con la cepa silvestre $\mathrm{H} 10407$ y con dos variantes de la cepa: una carente del plásmido LT (solo con el plásmido ST-CFA/I) y otra del plásmido ST-CFA/I (solo con el plásmido LT). Como era de esperar, los ratones silvestres eliminaron todas las cepas bacterianas a los tres días posinfección, mientras que los ratones $\mathrm{C} 57-\mathrm{cd}_{40 \mathrm{H}^{-}}$eliminaron la cepa que solo contenía al plásmido ST-CFA/I a los 31 días y se mantuvieron colonizados tanto con la cepa silvestre de ETEC, como con la cepa que solo contenía el plásmido LT hasta el día 113, cuando fueron sacrificados. ${ }^{18}$ Lo anterior sugirió que para la persistencia 
de $\mathrm{H} 10407$ en el ratón deficiente del ligando de CD40 es necesaria la presencia del plásmido LT, por lo que este modelo se puede utilizar para estudiar in vivo e in situ la interacción de cepas de ETEC aisladas de humanos.

\section{Prevalencia de EAEC}

EAEC es un patógeno que hasta el momento solo se ha identificado en humanos; se caracteriza por agruparse en forma de ladrillos apilados, ya sea sobre las células epiteliales 0 el cristal. ${ }^{31}$ Este patotipo fue aislado de un niño peruano con diarrea aguda. Con base en la presencia del regulador maestro aggR, descrito por primera vez en la cepa arquetipo 042 (serotipo O44:H18), el cual regula varios genes de patogenicidad, las EAEC se clasifican en típicas y atípicas (EAECt y EAECa). ${ }^{19,31}$ Las características clínicas de la enfermedad causada por EAEC se han descrito básicamente a partir de estudios en voluntarios adultos, brotes y casos esporádicos; estas cepas producen diarrea acuosa, frecuentemente con moco, con o sin sangre, fiebre, dolor abdominal, náuseas y vómito. ${ }^{3,19}$

Desde hace varios años se ha realizado la búsqueda de EAECt mediante la identificación del gen que codifica para el regulador transcripcional aggR; se ha identificado que esta bacteria se asocia con episodios de diarrea aguda y persistente en niños menores de cinco años, ${ }^{4-8,14,15}$ además, es el segundo agente más aislado de episodios de diarrea del viajero en adultos. ${ }^{21,23}$

En niños de países en desarrollo y subdesarrollados, la infección por EAEC se ha asociado con inflamación intestinal y desnutrición, así como con disminución en el crecimiento y función cognoscitiva. ${ }^{31-35}$ EAECt también se ha aislado en niños infectados con VIH de regiones menos desarrolladas. ${ }^{31}$ En México, EAEC se ha identificado en niños con diarrea que requirieron hospitalización, ${ }^{4,5,14}$ así como con diarrea de comuni$\mathrm{dad}^{15}$ (Tabla 1); recientemente fue asociada significativamente con diarrea aguda en niños menores de dos años en Sinaloa. ${ }^{15}$ Esta observación es importante porque estudios realizados en la última década en países subdesarrollados de Asia y África mostraron que la prevalencia de la infección por EAEC es similar en niños con diarrea y sin diarrea, ${ }^{9,10}$ lo que revela que la epidemiología de EAEC es diferente dependiendo de las poblaciones y sus condiciones socioeconómicas y que no se debe generalizar.

\section{Patogénesis de EAEC}

Varios estudios han sugerido que la patogénesis de EAEC se divide en tres etapas:

- La adherencia a la mucosa intestinal mediada principalmente por las fimbrias de adherencia agregativa.

- La liberación de enterotoxinas y citotoxinas.

- La inducción de la inflamación de la mucosa. ${ }^{31}$

El fenotipo característico de las EAEC, la adherencia agregativa, se debe a las fimbrias de adherencia agregativa, sin embargo, en diferentes cepas de EAEC también se ha asociado con otras adhesinas de tipo fimbriales y afimbriales. La heterogeneidad del reportorio de fimbrias es similar a la observada en los factores de colonización de ETEC. ${ }^{27,31}$ Las fimbrias de adherencia agregativa están codificadas en un plásmido de alto peso molecular, en el cual en la cepa 042, además del gen para la fimbria de adherencia agregativa II, también se encuentran los genes que codifican para varias moléculas involucradas en la patogénesis de esta bacteria, ${ }^{31}$ tal es el caso de las toxinas Pet (plasmid-encoded toxin), cuyo blanco es la alfa-fodrina y EAST-1, la toxina termoestable de EAEC, cuya actividad es similar a la de la toxina ST de ETEC, ya que también se une al receptor de la guanilato ciclasa sobre la membrana de los enterocitos del hospedero. Este plásmido también contiene los genes para la proteína críptica shf y aap (antiaggregative secreted protein), cuyos productos se requieren para la formación de la biopelícula característica de las EAEC y de una proteína que recubre a la bacteria para que esta se pueda dispersar a través del moco y llegar a la superficie de los enterocitos, respectivamente. ${ }^{31}$ De manera muy importante, en el mismo plásmido se encuentra codificado el gen agg $R$, cuyo producto es el regulador transcripcional maestro, el cual modula la expresión de todos los genes de las moléculas descritas.

EAEC produce otras toxinas con efecto citotóxico como las toxinas ShET1 (shigella enterotoxin 1) y Pic (protease involved in intestinal colonization), ambas codificadas en el cromosoma bacteriano. Hasta el momento no se han identificado moléculas asociadas exclusivamente con cepas de EAEC aisladas de casos de diarrea, por lo que la identificación de moléculas que caractericen cepas virulentas continúa siendo un área de gran interés científico. Se ha reportado que las cepas de EAEC aisladas de casos de diarrea formaban biopelículas más frecuentemente que las cepas aisladas de sujetos que no presentaban 
diarrea. ${ }^{36}$ El $67 \%$ de cepas de EAECt aisladas de casos severos de diarrea contienen los genes que codifican para las toxinas Pic y ShET1. ${ }^{14}$ Se ha mostrado que en los niños con infección persistente por EAEC se induce inflamación crónica intestinal en ausencia de diarrea, mientras que en las heces de sujetos con diarrea debida a EAECt se ha identificado lactoferrina y otros marcadores de inflamación. ${ }^{8,31}$ Por lo anterior se considera que la infección por EAEC induce inflamación en el intestino de niños y adultos, lo que resulta en daño al epitelio intestinal, disminuyendo la capacidad de absorción, que conlleva disminución en la absorción de nutrientes y, por ende, desnutrición, particularmente en niños de países subdesarrollados, ${ }^{8,31}$ manteniendo el círculo vicioso de diarrea y desnutrición.

\section{Modelos múridos de EAEC}

Debido a la falta de modelos animales de EAEC adecuados y accesibles, hasta el momento se desconoce la patogénesis de EAEC in vivo e in situ, es decir, los mecanismos que subyacen en su inicio, progresión y desenlace in vivo, como lo mencionaron Philipson et al. ${ }^{37}$ en su revisión de 2013, ya que en los modelos múridos reportados hasta ese momento siempre se eliminaba la microbiota local, fundamental en la homeostasis intestinal ${ }^{38}$ y no se había evaluado si la infección por EAEC inducía alteraciones in situ. Además, con estos modelos no se puede establecer si las alteraciones intestinales se deben a la infección por EAEC y no a la ausencia del microbiota local.

En 2010 se describió un modelo de infección en ratones $\mathrm{C} 57 \mathrm{BL} / 6$ adultos en el cual se analizaron dos cepas de referencia de EAEC, 042 y JM221, en el que los ratones antes de ser inoculados eran tratados con antibióticos, por lo que los autores consideraron que el modelo mostraba limitaciones. ${ }^{39}$ Posteriormente, ese mismo grupo desarrolló otro modelo de infección con EAEC, sin previo tratamiento con antibióticos con ratones $\mathrm{C} 57 \mathrm{BL} / 6$ recién destetados; se evaluó el efecto de la infección con EAEC 042 en ratones con desnutrición o que fueron previamente tratados con antibióticos (antes de ser inoculados). Observaron que ambos grupos de ratones presentaban incremento significativo en la infección por la bacteria, comparados con los ratones sin desnutrición que además no fueron tratados con antibióticos. ${ }^{40,41}$

Nuestro grupo de trabajo recientemente ha desarrollado un modelo de infección con EAEC 042 con ratones $\mathrm{C} 57 \mathrm{BL} / 6$ adultos y microbiota intacta, que ha permitido evaluar la interacción de estas bacterias con los enterocitos en los primeros días de infección. Hemos logrado establecer el papel de la fimbria de adherencia agregativa II en la infección del tracto intestinal utilizando una cepa silvestre y una mutante de la fimbria de adherencia agregativall; hemos observado que esta induce la movilización de beta catenina a través de su unión a la proteína MUC1, la cual está en la superficie del enterocito. ${ }^{42}$

Por otro lado, con un modelo de ratón BALB/C otro grupo de investigadores evaluó el efecto de dos cepas de EAEC aisladas de pacientes de la India con diarrea. ${ }^{8} \mathrm{~A}$ los 10 días posinoculación oral, los animales mostraron alteraciones en la superficie del íleon, ensanchamiento de la base de las microvellosidades, y en la lámina propia, infiltrados de células mononucleares, neutrófilos y algunos focos hemorrágicos. Sin embargo, los autores no mencionan si los animales fueron tratados con antibióticos previamente a la inoculación con estas bacterias, ni la dosis de bacterias que inocularon.

\section{Conclusiones}

En México, ETEC y EAEC son importantes agentes causales de diarrea en niños menores de cinco años. EAEC ocasiona diarrea en niños con desnutrición. La patogénesis y las moléculas responsables de la diarrea acuosa característica de ETEC son las toxinas LT y ST, las cuales están bien caracterizadas, mientras que en EAEC aún no están del todo identificadas ya que esta bacteria se define por la presencia de un regulador maestro que modula varios genes de virulencia. En la actualidad contamos con modelos múridos de infección con ETEC y EAEC con microbiota intacta, que permitirán entender la compleja interacción entre estos patógenos con su hospedero.

\section{Agradecimientos}

Para este trabajo, Diana Ríos Muñiz contó con el apoyo de la beca 20160973; Nadia Morán García de la beca 261884 y Mario Meza Segura de la beca 243144, todas del Conacyt. Durante este periodo, Teresa Estrada García dispuso del apoyo para el proyecto 254990, también por parte del Conacyt. Jorge Francisco Cerna Cortés es becario del programa Estímulos al Desempeño de los Investigadores y de la Comisión de Operación y Fomento de Actividades Académicas, ambos del Instituto Politécnico Nacional. 


\section{Bibliografía}

1. Secretaría de Salud. Anuario de morbilidad 2015. México: Dirección General de Epidemiologia/Secretaría de Salud; 2015.

2. Instituto Nacional de Estadística y Geografía. Estadística a propósito de día del niño (30 de abril). Datos nacionales. México: Instituto Naciona de Estadística y Geografía; 2018.

3. Sánchez-Uribe E, Esparza-Aguilar M, Parashar UD, Richardson V. Sustained reduction of childhood diarrhea-related mortality and hospitalizations in Mexico after rotavirus vaccine universalization. Clin Infect Dis. 2016;62:S133-S139.

4. Estrada-García T, Cerna JF, Paheco-Gil L, Velázquez RF, Ochoa TJ Torres J, et al. Drug-resistant diarrheogenic Escherichia coli, Mexico. Emerg Infect Dis. 2005:11:1306-1308.

5. Patzi-Vargas S, Zaidi M, Pérez-Martínez I, León-Cen M, Michel-Ayala A Chaussabel D, Estrada-García T. Diarrheagenic Escherichia coli carrying supplementary virulence genes are an important cause of moderate to severe diarrhoeal disease in Mexico. PLoS Negl Trop Dis. 2015; 9:e0003510.

6. Ochoa T, Ecker L, Barletta F, Mispireta M, Gil A, Contreras C, et al Age-related susceptibility to infection with diarrheagenic Escherichia coli among infants from periurban areas in Lima, Peru. Clin Infect Dis. 2009;49:1694-1702

7. Ochoa TJ, Mercado EH, Durand D, Rivera FP, Mosquito S, Contreras C, et al. Frequency and pathotypes of diarrheagenic Escherichia coli in Peruvian children with and without diarrhea. Rev Peru Med Exp Salud Publica. 2011;28:13-20

8. Saha DR, Guin S, Krishnan R, Nag D, Koley H, Shinoda S, Ramamurthy T. Inflammatory diarrhea due to enteroaggregative Escherichia coli: evidence from clinical and mice model studies. Gut Pathog. 2013;5:36.

9. Kotloff K, Nataro J, Blackwelder W, Nasrin D, Farag T, Panchalingam S, et al. Burden and aetiology of diarrhoeal disease in infants and young children in developing countries (the Global Enteric Multicenter Study, GEMS): a prospective, case-control study. Lancet. 2013:382:209-222.

10. Platts-Mills JA, Babji S, Bodhidatta L, Gratz J, Haque R, Havt A, et al. Pathogen-specific burdens of community diarrhoea in developing countries: a multisite birth cohort study (MAL-ED). Lancet Glob Health. 2015; 3:e564-e575.

11. Croxen MA, Law RJ, Scholz R, Keeney KM, Wlodarska M, Finlay BB Recent advances in understanding enteric pathogenic Escherichia coli. Clin Microbiol Rev. 2013:26:822-880.

12. Cerna JF, Nataro JP, Estrada-García T. Multiplex PCR for detection of three plasmid-borne genes of enteroaggregative Escherichia coli strains. J Clin Microbiol. 2003;41:2138-2140.

13. López-Saucedo C, Cerna JF, Villegas-Sepúlveda N, Thompson R, Velázquez FR, Torres J, et al. Single multiplex polymerase chain reaction to detect diverse loci associated with diarrheagenic Escherichia coli. Emerg Infect Dis. 2003:9:127-131.

14. Meza-Segura MA. Caracterización de los factores asociados con la severidad de la diarrea causada por los patogrupos de Escherichia coli. [Tesis doctoral]. México: Centro de Investigación y de Estudios Avanzados-Instituto Politécnico Nacional; 2017.

15. Canizalez-Román A, Flores-Villaseñor HM, González-Núñez E, Velázquez-Román J, Vidal JE, Muro-Amado S, et al. Surveillance of diarrheagenic Escherichia coli strains isolated from diarrhea cases from children, adults and elderly at northwest of Mexico. Front Microbiol. 2016:7:1924

16. Estrada-García T, López-Saucedo C, Thompson-Bonilla R, Abonce M, López-Hernández D, Santos JI, et al. Association of diarrheagenic Escherichia coli pathotypes with infection and diarrhea among Mexican children and association of atypical enteropathogenic E. coli with acute diarrhea. J Clin Microbiol. 2009;47:93-98.

17. Paniagua GL, Monroy E, García-González O, Alonso J, Negrete E, Vaca S. Two or more enteropathogens are associated with diarrhoea in Mexican children. Ann Clin Microbiol Antimicrob. 2007;28;6:17.

18. Bernal-Reynaga R, Thompson-Bonilla R, López-Saucedo C, Pech-Armenta M, Estrada-Parra S, Estrada-García T. C57-CD40 ligand deficient mice: a potential model for enterotoxigenic Escherichia coli $(\mathrm{H} 10407)$ colonization. Vet Immunol Immunopathol. 2013;152:50-56.

19. Estrada-García T, Pérez-Martínez I, Bernal-Reynaga R, Zaidi M. Enteroaggregative Escherichia coli: a pathogen bridging the north and south. Curr Trop Med Rep. 2014;2:88-96

20. Hosangadi D, Smith PG, Kaslow DC, Giersing BK, WHO ETEC \& Shigella Vaccine Consultation Expert Group. WHO consultation on ETEC and Shigella burden of disease, Geneva, 6-7 $7^{\text {th }}$ April 2017: Meeting report. Vaccine. 2018;pii: S0264.

21. Jiang ZD, DuPont HL. Etiology of travellers' diarrhea. J Travel Med. 2017;24:S13-S16

22. Steffen R, Hill DR, DuPont HL. Traveler's diarrhea: a clinical review. JAMA. 2015;313:71-80.

23. Paredes-Paredes M, Okhuysen PC, Flores J, Mohamed JA, Padda RS, Gonzalez-Estrada A, et al. Seasonality of diarrheagenic Escherichia coli pathotypes in the US students acquiring diarrhea in Mexico. J Travel Med. 2011;18:121-125

24. Bolick DT, Medeiros P, Ledwaba SE, Lima AA, Nataro JP, Barry EM, et al. Critical role of zinc in a new murine model of enterotoxigenic Escherichia coli diarrhea. Infect Immun. 2018;86:e00183

25. Fleckenstein J, Sheikh A, Qadri F. Novel antigens for enterotoxigenic Escherichia coli vaccines. Expert Rev Vaccines. 2014;13:631-639.

26. Sahl JW, Sistrunk JR, Baby NI, Begum Y, Luo Q, Sheikh A, et al. Insights into enterotoxigenic Escherichia coli diversity in Bangladesh utilizing genomic epidemiology. Sci Rep. 2017;7:3402.

27. Madhavan TP, Sakellaris H. Colonization factors of enterotoxigenic Escherichia coli. Adv Appl Microbiol. 2015;90:155-197.

28. $\mathrm{Ng} \mathrm{KM}$, Ferreyra JA, Higginbottom SK, Lynch JB, Kashyap PC Gopinath S, et al. Microbiota-liberated host sugars facilitate post-antibiotic expansion of enteric pathogens. Nature. 2013;502:96-99.

29. Parker KD, Albeke SE, Gigley JP, Goldstein AM, Ward NL. Microbiome composition in both wild-type and disease model mice is heavily influenced by mouse facility. Front Microbiol. 2018:9:1598.

30. Allen KP, Randolph MM, Fleckenstein JM. Importance of heat-labile enterotoxin in colonization of the adult mouse small intestine by human enterotoxigenic Escherichia coli strains. Infect Immun. 2006;74:869-875.

31. Estrada-García T, Navarro-García F. Enteroaggregative Escherichia coli pathotype: a genetically heterogeneous emerging foodborne enteropathogen. FEMS Immunol Med Microbiol. 2012;66:281-298.

32. Guerrant RL, Oriá RB, Moore SR, Oriá MO, Lima AA. Malnutrition as an enteric infectious disease with long-term effects on child development. Nutr Rev. 2008;66:487-505.

33. Opintan JA, Newman MJ, Ayeh-Kumi PF, Affrim R, Gepi-Attee R, Sevilleja JE, et al. Pediatric diarrhea in southern Ghana: etiology and association with intestinal inflammation and malnutrition. Am J Trop Med Hyg. 2010;83:936-943.

34. Acosta GJ, Vigo N, Durand D, Riveros M, Arango S, Zambruni M, et al. Diarrheagenic Escherichia coli: prevalence and pathotype distribution in children from peruvian rural communities. Am J Trop Med Hyg. 2016:95:574-579.

35. Rogawski E, Guerrant R, Havt A, Lima I, Medeiros P, Seidman J, et al. Epidemiology of enteroaggregative Escherichia coli infections and associated outcomes in the MAL-ED birth cohort. PloS Negl Trop Dis. 2017:11:e0005798.

36. Mohamed JA, Huang DB, Jiang ZD, DuPont HL, Nataro JP, Belkind-Gerson $\mathrm{J}$, et al. Association of putative enteroaggregative Escherichia col virulence genes and biofilm production in isolates from travelers to developing countries. J Clin Microbiol. 2007;45:121-126.

37. Philipson WC, Bassaganya-Riera J, Hontecillas R. Animal models of enteroaggregative Escherichia coli infection. Gut Microbes. 2013. 4:281-291.

38. Koboziev L, Reinoso-Webb C, Furr K L, Grisham MB. Role of the enteric microbiota in intestinal homeostasis and inflammation. Free Radic Biol Med. 2014:68:122-133.

39. Roche JK, Cabel A, Sevilleja J, Nataro J, Guerrant RL. Enteroaggregative Escherichia coli (EAEC) impairs growth while malnutrition worsens EAEC infection: a novel murine model of the infection malnutrition cycle. J Infect Dis. 2010;202:506-514

40. Bolick DT, Roche JK, Hontecillas R, Bassaganya-Riera J, Nataro JP, Guerrant RL. Enteroaggregative Escherichia coli strain in a novel weaned mouse model: exacerbation by malnutrition, biofilm as a virulence factor and treatment by nitazoxanide. J Medical Microbiol. 2013;62:896-905.

41. Medeiros P, Bolick DT, Roche JK, Noronha F, Pinheiro C, Kolling GL, et al. The micronutrient zinc inhibits EAEC strain 042 adherence, biofilm formation, virulence gene expression, and epithelial cytokine responses benefiting the infected host. Virulence. 2013;4:624-633.

42. Morán-García NE. Desarrollo y caracterización de un modelo múrido de infección con Escherichia coli enteroagregativa 042 y la participación de la fimbria AAF/II en la infección [Tesis doctoral]. México: Centro de Investigación y de Estudios Avanzados-Instituto Politécnico Nacional; 2018. 